[Agr. Biol. Chem., Vol. 33, No. 6, p. 818 825, 1969]

\title{
Flavonols in the Pollen of Tea Flowers
}

\section{Part I. Occurrence of Pollenitin and Pollenin a, b, and c}

\author{
By Yutaka SAKAмото \\ Tea Research Station, Ministry of Agriculture and Forestry, \\ Kanaya, Shizuoka \\ Received September 2, 1968
}

\begin{abstract}
A new flavonol and its glycosides were isolated from the pollen of tea flowers. Their UV, IR, MS, and NMR spectra, alkaline degradation and color reactions have supported that the chemical structure of the flavonol is 3,5,8,4'-tetrahydroxy-7-methoxyflavone and that two glycosides were its 3-rhamnoglucoside and 3-monoglucoside, respectively. But another glycoside remained unidentified as to the sugar moiety. This flavonol, its rhamnoglucoside, monoglucoside and undetermined glycoside were named pollenitin, pollenin $a, b$, and $c$, respectively.
\end{abstract}

Flavonol glycosides in the pollen of Crocus have been reported to function as sexual substances $^{11}$ on the green algae Chlamydomonas. Rutin and quercitrin in the pollen of Forsythia which is selfsterile are known to be inhibitors of fertilization. ${ }^{21}$

Tea plants are generally selfsterile and there is difference in the affinity for fertilization among the varieties of tea. However, in the previous study, flavonol glycosides in the pollen of tea flowers were found not to be hydrolyzed with the acetone powder prepared from the pistiles of tea flowers, indicating that they were unlikely to be involved in the mechanism of fertilization of tea flowers under the same condition as Forsythia.

In every variety, four flavonol spots have been detected on the two-dimensional paper chromatograms of the aqueous methanol extract of tea pollens. The $R f$ values are shown in Table I. Pigment D was precipitated with neutral lead acetate and the others were precipitated with ammonium hydroxide from the filtrate after removal of neutral lead acetate salt. After final purification by preparative paper chromatography, pigments $\mathrm{A}$ and $\mathrm{D}$ were isolated as yellow thin plate crystals, and $\mathrm{B}$ and $\mathrm{C}$ were obtained as yellow powders in small quantities.

On acid hydrolysis, pigment A gave rham-

Table 1. Paper Chromatographic Data of Tea pollen flavonoids

Color on paper with

\begin{tabular}{cccc} 
Pigments & \multicolumn{3}{c}{$R f$ with } \\
A & 0.38 & $75 \%$ PhOH & $2 \%$ AcOH \\
B & 0.51 & 0.75 & 0.72 \\
C & 0.43 & 0.55 & 0.33 \\
D & 0.87 & 0.75 & 0.47
\end{tabular}

\begin{tabular}{|c|c|c|c|c|c|c|}
\hline \multirow[b]{2}{*}{$\begin{array}{l}\text { vis. } \\
\mathrm{Y}\end{array}$} & \multirow[b]{2}{*}{$\begin{array}{l}\text { uv } \\
\text { DY }\end{array}$} & \multicolumn{2}{|c|}{$\mathrm{NH}_{3}$} & \multicolumn{2}{|c|}{$\mathrm{AlCl}_{3}$} & \multirow{2}{*}{$\begin{array}{c}\mathrm{FeCl} \\
\text { vis. } \\
\mathrm{YBr}\end{array}$} \\
\hline & & $\begin{array}{l}\text { vis. } \\
\text { Y }\end{array}$ & $\begin{array}{c}\mathrm{uv} \\
\mathrm{d}-\mathrm{Y}\end{array}$ & $\begin{array}{l}\text { vis. } \\
\text { Y }\end{array}$ & $\begin{array}{c}\text { uv } \\
\mathrm{GrY}\end{array}$ & \\
\hline$Y$ & $\overline{D Y}$ & $\bar{Y}$ & $\mathrm{~d}-\mathrm{Y}$ & $Y$ & GrY & $\mathrm{YBr}$ \\
\hline Y & DY & $Y$ & $d-Y$ & $Y$ & GrY & $\mathrm{YBr}$ \\
\hline Y & $d-Y$ & $Y$ & Y & $\mathrm{Y}$ & $\mathrm{Y}$ & $\mathrm{YBr}$ \\
\hline
\end{tabular}

BAW: $n$-Butanol: acetic acid: $\mathrm{H}_{2} \mathrm{O}=4-1-2$ vol $\%$

Y: yellow Gr: green DY: dark yellow d-Y: dull yellow Br: brown

1) R. Kuhn u. I. Löw, Ber., 77B, 196, 202 (1944).

2) R. Kuhn u. I. Löw, ibid., 82, 474 (1949). 


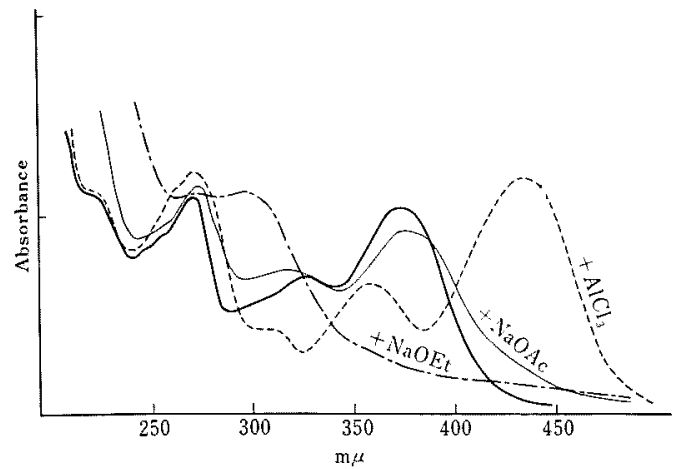

FIG. 1. UV Spectra of Pollenitin (in EtOH)

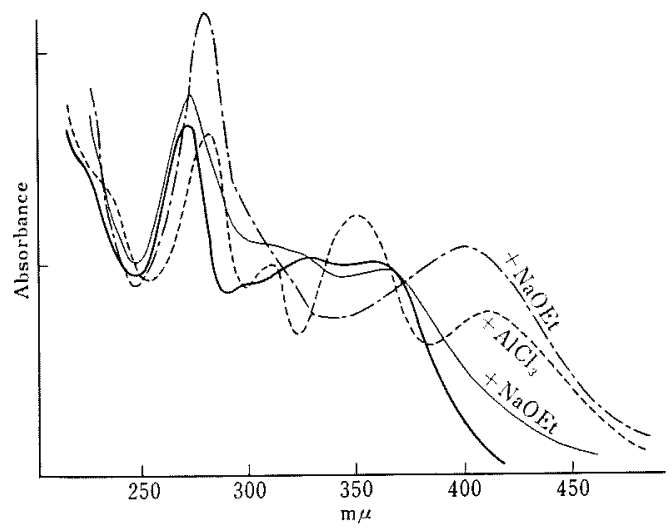

FIG. 2. UV Spectra of Pollenin a (in EtOH)

nose, glucose (about equal mole estimated from the spots) and pigment $\mathrm{D}$, pigment $\mathrm{B}$ also gave glucose and pigment $\mathrm{D}$, but the sugar moiety of pigment $C$ could not be identified, since it was isolated in very small amount. However, pigment $\mathrm{C}$ also gave pigment $\mathrm{D}$ as its aglycone. Accordingly, pigment A was found to be rhamnoglucoside (The sequence of sugars was not determined in this work), and $\mathrm{B}$ to be monoglucoside of pigment D. Though pigment $\mathrm{C}$ seemed to be a glycoside of pigment $\mathrm{D}$, its sugar moiety was unknown yet.

The absorption spectral data on the aluminum chloride complex-, sodium acetate-, sodium ethylate-shifts, and methyl ethers indicated that they belong to flavonols, that there have been free hydroxyl groups in 3, 5, 4'positions and a methoxyl group in 7-position in pigment $\mathrm{D}$ (aglycone), and that the sugar components of both pigments $\mathrm{A}$ and $\mathrm{B}$ were to be combined with the hydroxyl group in 3-position (Fig. 1 and Fig. 2).

As shown in mass spectrum (Fig. 3) pigment $\mathrm{D}$ has the parent molecular ion at $m / e=$ 316 , and $(M-15)^{+}$which is refered to the loss of a methyl group, was observed at $m / e=301$ as the base peak. This cracking pattern was also observed in the mass spectrum of acacetin* Therefore, the molecular weight of pigment $\mathrm{D}$ is believed to be 316 . From the spectral studies it has been assumed that pigment $\mathrm{D}$ is $3,5,4^{\prime}$-trihydroxy-7-methoxyflavone derivative ( $M W=300$ ). Accordingly, the difference of 16 in the molecular weight indicates the presence of a hydroxyl group in the A-ring.

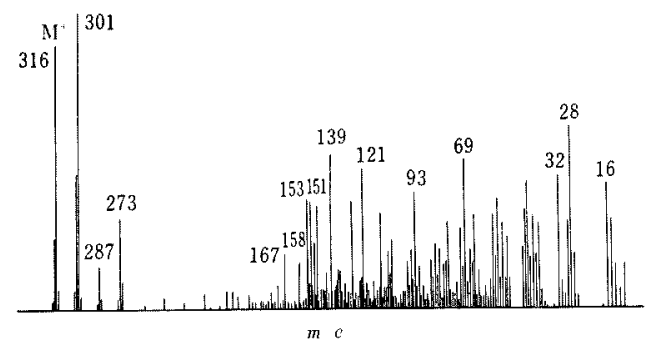

FIG. 3. Mass Spectrum of Pollenitin.

NMR analysis of pigment $\mathrm{D}$ was done by first converting it into the trimethylsilyl ether. In the resultant spectrum of this derivative, four proton signals displayed the typical signal pattern for B-ring which was substituted only at $4^{\prime}$-position: 2 protons of $2^{\prime}$ and $6^{\prime}$, doublet, at 7.97 and $8.12 \mathrm{ppm}$.; 2 protons of $3^{\prime}$ and $5^{\prime}$, doublet, at 6.78 and $6.93 \mathrm{ppm}$. The $\mathrm{C}-5$ and C-7 protons of the A-ring of the flavonoid give rise to signals in the slightly lower field than $7 \mathrm{ppm}^{.5 \sim 8 !}$ Furthermore, a singlet near $6.17 \mathrm{ppm}$, integrating for one proton, occurs

* unpublished work. 


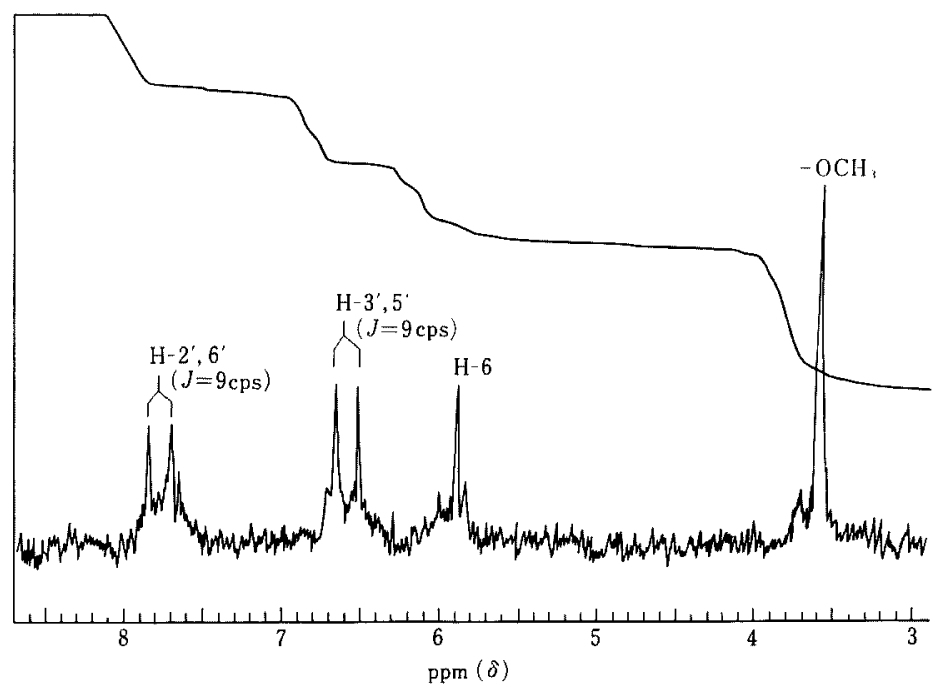

FIG. 4. MNR Spectrum of Trimethylsilylated Pollenitin in $\mathrm{CGI}_{4}$ (60 Mc. Internal St.=TMS)

in the region expected for either $\mathrm{C}-6$ or $\mathrm{C}-8$ proton of the A-ring. Only one A-ring proton is observed in each of the spectra of vitexin $(\mathrm{H}-6,6.29 \mathrm{ppm})$, isovitexin $(\mathrm{H}-8,6.56 \mathrm{ppm})$, orientin (H-6, $6.30 \mathrm{ppm})$, and isoorientin $(\mathrm{H}-$ $8,6.56 \mathrm{ppm})$. In each case, it appears as a singlet indicating the absence of a coupled meta-proton, and H-6 appears at the higher field than $\mathrm{H}-8 .^{81}$ Accordingly, a singlet near $6.17 \mathrm{ppm}$ in the spectrum of silylated pigment $\mathrm{D}$ is attributed to the $\mathrm{C}-6$ proton of the Aring, since it is in the relatively higher field at the region of $6.0 \sim 6.7 \mathrm{ppm}$. If the silyl ether of the flavonoid is kept being exposed to the atmosphere, the silyl group at $\mathrm{C}-5$ is occasionally hydrolyzed." In the NMR spectrum of silylated pigment $D$, the signal arising from the proton of $\mathrm{C}-5$ hydroxyl group was also observed in the downfield near 11.6 $\mathrm{ppm}$, indicating that a free hydroxyl group was present in 5-position, this is supported by aluminum chloride shifts in the UV spectra. Moreover, a singlet near $3.85 \mathrm{ppm}$ for three protons indicated the presence of a methoxyl group. In alkaline degradation of the pigment, however, iretol (1,3,5-trihydroxy-2-meth- oxybenzene) was not detected. Consequently, the methoxyl group seemed not to be located at either 6- or 8-positions, but be located at 7-position. As described later, it was revealed in the spectral studies that a 7-hydroxyl group seemed to be protected and that a free o-dihydroxyl group was not present. These results also indicate that a methoxyl group must be located at 7-position.

The infrared spectrum of pigment $\mathrm{D}$ showed intense absorptions in the region of $3400 \mathrm{~cm}^{-1}$ indicating the presence of many hydroxyl groups and a characteristic absorption in the

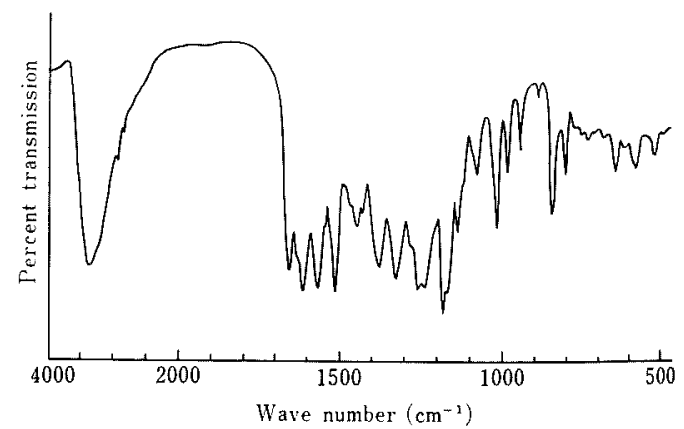

FIG. 5. IR Spectrum of Pollenitin ( $\mathrm{KBr}$ disc). 


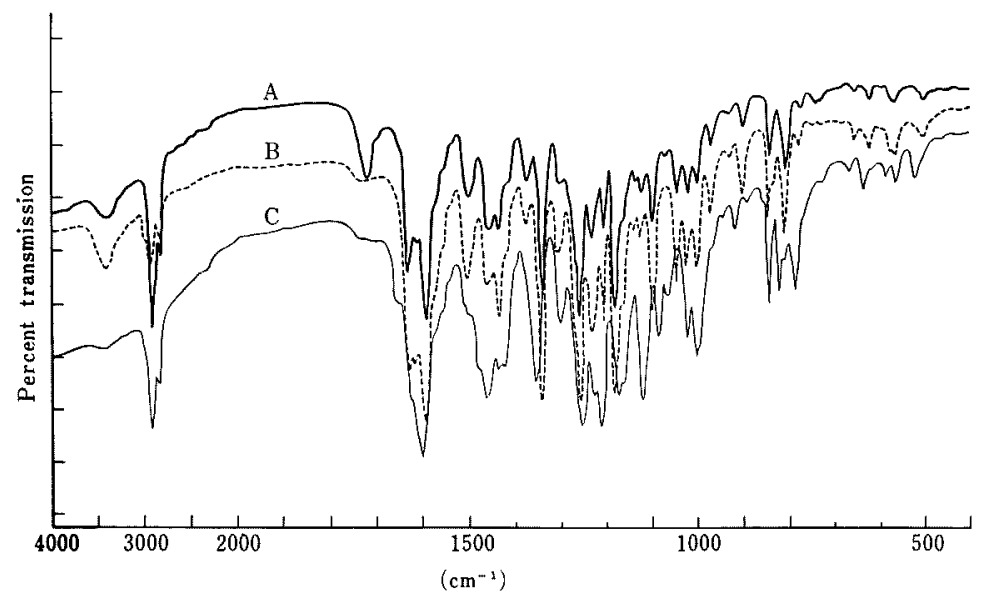

FIG. 6. IR Spectra of Methyl Pollenitin, Pentamethyl Herbacetin and Dimethyl Mikanin ( $\mathrm{KBr}$ disc).

A: Methyl pollenitin. B: Pentamethyl herbacetin (5,7,8,3,4'-pentamethoxyflavone). C: Dimethyl mikanin (5,6,7,3,4'-pentamethoxyflavone).

region of $1650 \mathrm{~cm}^{-1}$ for the carbonyl group (Fig. 5).

Hydrolytic fission of pigment $\mathrm{D}$ gave $p$ hydroxybenzoic acid, but the phenolic substance derived from the A-ring could not be determined. It was not identical with iretol obtained as an alkaline degradation product of wogonin. ${ }^{31 *}$ These results showed that the B-ring of the pigment has a hydroxyl group only at $4^{\prime}$-position.

In order to decided which position, 6 or 8 , of the A-ring is substituted with a hydroxyl group, though it was supposed to be 8-position from the data of the NMR spectrum, the demethylated substance of pigment $D$ was compared with $5,6,7,3,4^{\prime}$-pentahydroxyflavone and $5,7,8,3,4^{\prime}$-pentahydroxyflavone by examination of their paper chromatograms, and moreover methyl derivatives of pigment $\mathrm{D}$, $5,6,7,3,4^{\prime}$-, and 5,7,8,3,4'-pentamethoxyflavones were compared with one another by IR analysis. The demethylated substance ob-

3) S. Hattori and K. Hayashi, Nippon Kagaku Kaishi, 54, 919 (1933).

* Wogonin, used in this work, was isolated from Scutellaria baicalensis Gorgi by Dr. S. Kuwatsuka. tained by the treatment of pigment $\mathrm{D}$ with hydroiodic acid was not identical with $5,6,7,3$, $4^{\prime}$-pentahydroxyflavone prepared by demethylation of mikanin ${ }^{* *}\left(3,5\right.$-dihydroxy-6,7, $4^{\prime}$ trimethoxyflavone), but it was identical with herbacetin ${ }^{* *}\left(5,7,8,3,4^{\prime}\right.$-pentahydroxyflavone) in the $R f$ value and color of the spot, which gave characteristic blue color by spraying sodium carbonate solution. Moreover, it was found that the methyl derivative prepared by methylation of pigment $\mathrm{D}$ was not identical with $5,6,7,3,4^{\prime}$-pentamethoxyflavone $(3,5$-dimethyl mikanin), but it was identical with pentamethyl herbacetin ${ }^{* *}\left(\mathrm{mp} 158^{\circ} \mathrm{C}\right)$ as the result of IR analysis. (Fig. 6).

Both alcoholic solutions of pigment $\mathrm{A}$ and $D$ did not give characteristic brownish red color, but its color intensified with $p$-benzoquinone alcoholic solution. However, each of them gave brown color with the reagent after allowing the mixture to stand at room temperature over night. On the contrary, herb-

** The author expresses his thanks to Dr. T. Nakabayashi, Shizuoka Univ,, for gift of these authentic samples and also to Dr. K. Y.Sim, Singapore Univ., for authentic mikanin. 
acetin gave deep red color immediately after the reagent was added, but the color faded into yellow on standing over night.

Although a positive result was not obtained in the gossypeton reaction for pollen flavonoids, chemical structures as shown in Fig. 7 are assigned to them from the above-mentioned results, and the molecular formula of pigment $\mathrm{D}$ is proposed to be $\mathrm{C}_{16} \mathrm{H}_{12} \mathrm{O}_{7}$ (MW= 316).

There are many references about the isolation or synthesis of $3,5,7,8,4^{\prime}$-pentahydroxyflavone derivatives, but flavonol and its glycosides having such chemical structures as shown in Fig. 7, that is 3,5,8,4'-tetrahydroxy-7-methoxyflavones, have not been reported.

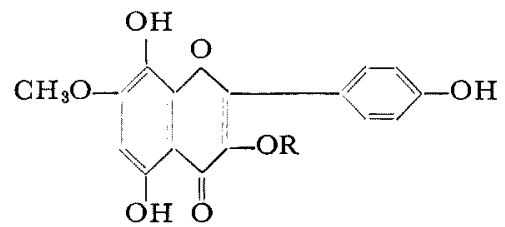

Pigment A: $\mathrm{R}=$ rhamnoglucosyl residue Pigment B: $\mathrm{R}=$ glucosyl residue

Pigment $\mathrm{D}: \mathrm{R}=\mathrm{H}$

Fig. 7. The Chemical Structures of Pigments A, $\mathrm{B}$, and $\mathrm{D}$.

The author proposes to name this new flavonol, pigment D, "Pollenitin" and its glycosides, pigment $\mathrm{A}, \mathrm{B}$, and $\mathrm{C}$, "Pollenin $a, b$, and $c$," respectively.

\section{EXPERIMENTAL}

Isolation of the pigments from the pollen of tea flowers. Fresh pollens $(330 \mathrm{~g})$ of tea flowers were extracted with hot aqueous methanol and the extract was condensed to a syrup under reduced pressure. After addition of water to the syrup, the aqueous solution was shaken three times with chloroform and the chloroform soluble fractions were separated off. At first, neutral lead acetate solution was added to the aqueous solution free from chloroform. The precipitates thus obtained were washed with distilled water, and then decomposed with $10 \%$ sulfuric acid. After removal of lead sulfate, the filtrate was extracted with ethyl ether several times. The ethyl ether soluble fractions were combined together, dried with sodium sulfate, and then the solvent was evaporated off. The residue was washed with benzol and dissolved in a small quantity of methanol. A large quantity of water added into the methanol solution and again ether extraction was repeated. The yellow residue was finally dissolved in hot aqueous ethanol, treated with active carbon, and then filtered. After concentrated and refrigerated for one day, lemon yellow crystals thereby produced were filtered and dried in a desiccator. Recrystallizations from $50 \%$ aqueous ethanol were repeated and pure pollenitin was obtained (about $15 \mathrm{mg}$ ).

The filtrate from neutral lead acetate precipitates was treated with ammonium hydroxide and lead acetate solution. The yellow precipitates formed thereby were filtered by suction, washed with dist. water and decomposed with hydrogen sulfide, suspending in methanol. After removal of black lead sulfide by suction, the filtrate was concentrated under reduced pressure and treated with dist. water. The aqueous solution was extracted at first with ethyl ether and

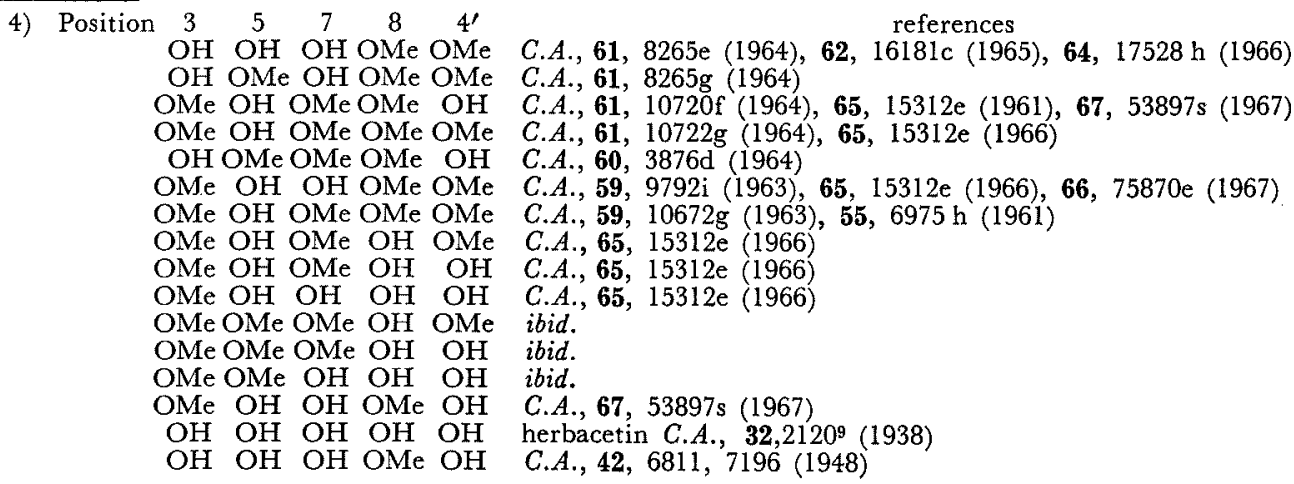


then with $n$-butanol several times. The $n$-butanol extracts were concentrated and dried in vacuo. The residue was dissolved in a small quantity of methanol, and ethyl ether was poured into it until no more precipitates were formed. (The yield of crude pollenin were about $600 \mathrm{mg}$ ). Repeating several times the above procedure, purified pollenin was obtained as an amorphous yellow substance (about $450 \mathrm{mg}$ ).

The polletin mixture was then separated into three components $a, b$, and $c$ by preparative paper chromatography by using Whatman $3 \mathrm{MM}$. The band of each pigment, detected under ultraviolet ray, was separately eluted from the filter paper with $30 \%$ aqueous methanol and dried under reduced pressure. Thus, pollenin a was obtained as yellow thin plate crystals after recrystallization, and pollenin $b$ and $c$ were obtained in a small quantity as yellow powder, by drying each elute in vacuo, but the amount of $\mathrm{c}$ was so small that it was only tested by paper chromatography in this study.

\section{General properties of the pigments}

Pollenin a: Yellow fine crystals. Thin plates (microscopically). Mp $154 \sim 155^{\circ} \mathrm{C}$. Very soluble in water. Soluble in methanol and hot ethanol, but insoluble in ethyl acetate and ethyl ether. Reduction of its alcoholic solution with magnesium and conc. hydrochloric acid gave pink-red color and with zinc and conc. hydrochloric acid gave orange-red color.
Pollenitin: Lemon yellow fine crystals. Thin plates (microscopically). $\mathrm{Mp} 248 \sim 250^{\circ} \mathrm{C}$ (decomp.). Soluble in ethanol, methanol, ethyl ether, hot water, glacial acetic acid and pyridine. Insoluble in cold water. $\mathrm{FeCl}_{3}$ reaction: dirty green, changed to yellowish brown. Its alcoholic solution gave pink-red color with magnesium and conc. hydrochloric acid. Alcoholic solution of pollenitin gave no color with $p$-benzoquinone which was prepared from hydroquinone by sodium dichromate and conc. sulfuric acid (gossypeton color reaction). The results of the color reactions of these pigments were shown in Table I.

Acetyl derivative of pollenitin. Colorless long needles. $\operatorname{mp} 157 \sim 158^{\circ} \mathrm{C}$.

Methylation and demethylation of pollenitin. Methylation of pollenitin and mikanin was carried out by the usual method with a great excess of diazomethane in ethyl ether or dimethyl sulfate and potassium carbonate in anhydrous acetone. Demethylation of pollenitin, mikanin, and tetramethyl herbacetin was carried out by the usual method with hydroiodic acid ( $\mathrm{SG}=1.47)$ and a small quantity of phenol for $2 \mathrm{hr}$ at $150^{\circ} \mathrm{C}$. $R f$ values and colors of these substances were shown in Table II.

Hydrolysis of the pigments. By boiling under reflux with $5 \%$ hydrochloric acid for about an hour, or by heating directly on fire with $2 \%$ sulfuric acid

Table II. Rf VAlUes and Colors of Methyl- and DemethylDerivatives of Pollenitin

\begin{tabular}{ccccc} 
& \multicolumn{4}{c}{ Solvents } \\
\cline { 5 - 6 } Sub. & I & II & III & IV \\
1 & 0.33 & 0.99 & 0.76 & 0.91 \\
2 & 0.75 & 0.99 & 0.96 & 0.98 \\
3 & 0.26 & - & - & - \\
4 & 0.26 & 0.99 & 0.32 & 0.73 \\
5 & 0.69 & 0.99 & 0.99 & 0.99 \\
6 & 0.75 & 0.99 & - & - \\
7 & 0.25 & 0.99 & 0.32 & 0.73 \\
8 & 0.53 & 0.99 & 0.95 & 0.98 \\
9 & 10.35 & - & - & -
\end{tabular}

\begin{tabular}{cccc}
\multicolumn{4}{c}{ Colors } \\
\cline { 2 - 4 } uv & $\mathrm{Na}_{2} \mathrm{CO}_{3}$ & uv/Na $\mathrm{Na}_{2} \mathrm{CO}_{3}$ & $\mathrm{AlCl}_{3}$ \\
$\mathrm{YO}$ & $\mathrm{Y}$ & $\mathrm{DY}$ & $\mathrm{LY}$ \\
$\mathrm{LY}$ & $\mathrm{P}-\mathrm{Y}$ & $\mathrm{LY}$ & $\mathrm{p}-\mathrm{YO}$ \\
$\mathrm{D}$ & Blue & $\mathrm{D}$ & $\mathrm{p}-\mathrm{YO}$ \\
$\mathrm{D}$ & Blue & $\mathrm{D}$ & $\mathrm{YO}$ \\
$\mathrm{D}$ & $\mathrm{p}-\mathrm{Y}$ & $\mathrm{D}$ & $\mathrm{Y}$ \\
$\mathrm{LY}$ & $\mathrm{P}-\mathrm{Y}$ & $\mathrm{LY}$ & $\mathrm{p}-\mathrm{Y}$ \\
$\mathrm{D}$ & $\mathrm{Y}$ & $\mathrm{GrY}$ & $\mathrm{p}-\mathrm{Y}$ \\
$\mathrm{Y}$ & $\mathrm{Y}$ & $\mathrm{GrY}$ & $\mathrm{LY}$ \\
$\mathrm{D}$ & $\mathrm{Y}$ & $\mathrm{Y}$ & $\mathrm{LY}$
\end{tabular}

Sub. 1: pollenitin, 2: methyl deriv. of pollenitin, 3: demethyl deriv. of pollenitin, 4: herbacetin, 5: tetramethyl herbacetin, 6: pentamethyl herbacetin, 7: demethyl deriv. of tetramethyl herbacetin, 8: mikanin, 9: demethyl deriv. of mikanin.

Solv. I: $50 \%$ acetic acid, II: ethyl acetate saturated with $\mathrm{H}_{2} \mathrm{O}$, III: $75 \%$ phenol, IV: $n$-butanol:acetic acid: water $=4: 1: 2$ vol. $\%$.

Color Y: yellow, O: orange, p-: pale, LY: lemon yellow, D: dark, Gr: green. 
Table III. Ultraviolet Extinctions of the Pigments Isolated FROM THE POLLEN OF TEA FLOWERS

\begin{tabular}{|c|c|c|c|c|c|}
\hline & Pollenitin & $\begin{array}{l}\text { Methyl deriv : of } \\
\text { pollenitin with } \\
\mathrm{CH}_{2} \mathrm{~N}_{2}\end{array}$ & $\begin{array}{l}\text { Pollenin } \\
\text { a }\end{array}$ & $\begin{array}{l}\text { Methyl deriv. of } \\
\text { pollenin a with } \\
\mathrm{CH}_{2} \mathrm{~N}_{2}\end{array}$ & $\begin{array}{l}\text { Pollenin } \\
\text { b }\end{array}$ \\
\hline & $\begin{array}{l}273^{\mathrm{m} \mu} \\
326 \\
377\end{array}$ & $\begin{array}{l}{ }_{272}^{\mathrm{m} \mu} \\
323 \\
355 \sim 6\end{array}$ & $\begin{array}{l}{ }_{273}^{\mathrm{m} \mu} \\
327 \\
359\end{array}$ & $\begin{array}{l}\quad \mathrm{m} \mu \\
272 \\
321 \sim 2 \\
354 \sim 5\end{array}$ & $\begin{array}{l}\quad \mathrm{m} \mu \\
273 \sim 4 \\
333 \\
358 \sim 9\end{array}$ \\
\hline$+\mathrm{AlCl}_{3}$ & $\left\{\begin{array}{l}272 \sim 3 \\
307 \\
357 \\
434\end{array}\right.$ & $\begin{array}{l}281 \\
312 \\
346 \\
414\end{array}$ & $\begin{array}{l}283 \\
311 \\
351 \\
414\end{array}$ & $\begin{array}{l}280 \\
312 \\
345 \\
415\end{array}$ & $\begin{array}{l}283 \\
311 \\
351 \\
412\end{array}$ \\
\hline$+\mathrm{NaOAc}$ & $\left\{\begin{array}{l}274 \\
320 \\
380\end{array}\right.$ & $\begin{array}{l}273 \\
320 \\
355\end{array}$ & $\begin{array}{l}273 \\
328 \sim 9 \\
358 \sim 9\end{array}$ & $\begin{array}{l}272 \\
322 \\
356\end{array}$ & $\begin{array}{l}275 \sim 6 \\
310 \\
343\end{array}$ \\
\hline$+\mathrm{NaOEt}$ & decomp. & $\begin{array}{l}272 \\
355\end{array}$ & $\begin{array}{l}284 \\
400\end{array}$ & & $\begin{array}{l}285 \\
311^{\text {sh }} \\
392 \sim 3\end{array}$ \\
\hline $\begin{array}{c}+\mathrm{NaOAc}_{3} \mathrm{BO}_{3} \\
\mathrm{H}_{2} \mathrm{BO}\end{array}$ & $\left\{\begin{array}{l}273 \\
320 \\
378\end{array}\right.$ & & & & \\
\hline
\end{tabular}

about five minutes, these pigments were hydrolyzed. After filtration of each aglycone by suction, the hydrolyzate was dried in a vacuum desiccator with sodium hydroxide (HCI-hydrolysis), or was neutralized with barium carbonate, filtered by suction, and concentrated to a syrup $\left(\mathrm{H}_{2} \mathrm{SO}_{4}\right.$-hydrolysis). The aglycones and sugar components were examined by a comparison of their $R f$ values with those of authentic samples, using five different developing solvents and color reagents. Examination of the paper chromatograms revealed that their aglycones were all identical with pigment $\mathrm{D}$ (pollenitin) and that the sugar components of pollenin $a$ and $b$ were identical with rhamnose and glucose (about equal mole estimated from the spots), and glucose, respectively, but pollenin $c$ was so small in quantity that the sugar moiety could not be confirmed. Accordingly pollenin a and $\mathrm{b}$ were believed to be pollenitin rhamnoglucoside and pollenitin monoglucoside, respectively.

Ultraviolet spectra of the pigments.* The ultraviolet spectra of the pigments were examined with a Beckman DB spectrophotometer with a potentiometric recorder. UV extinctions of the pigments were shown in Table III, Fig. 1 and Fig. 2.

Pollenitin: Pollenitin, the aglycone of pollenin $a$, $\mathrm{b}$ and $\mathrm{c}$ exhibited strong absorptions at $377 \mathrm{~m} \mu$ ( $\varepsilon$,

* T. A. Geissman, "The Chemistry of Flavonoid Compounds," Pergamon Press, 1962, p. $107 \sim 131$.
15,570) (Band I) and $273 \mathrm{~m} \mu(\varepsilon, 16,662)$ (Band II), and a weak absorption at $326 \mathrm{~m} \mu$, indicating that it belongs to flavonol. That is, it has a free hydroxyl group in 3-position. Band I bathochromically shifted about $57 \mathrm{~m} \mu$ on the addition of aluminum chloride and the spectral curve was similar to that of the typical aluminum-flavonol complex. The presence of a free hydroxyl group in 5-position was also confirmed by the bathochromic shift (about $9 \mathrm{~m} \mu$ ) of Band II of 3-0-methylpollenitin due to the addition of aluminum chloride. Fused sodium acetate gave a very small shift in the position of the peak of Band II, so that the hydroxyl group in 7-position seemed to be protected with the methyl group. (The presence of a methoxyl group has been confirmed in the spectra of NMR and MS). Alcoholic solution of pollenitin was decomposed by addition of sodium ethylate within several minutes. This indicates that both of the hydroxyl groups in 3- and $4^{\prime}$-positions could be free. Boric acid-sodium acetate did not significantly alter the spectrum of pollenitin. Accordingly, free 0 dihydroxyl groups are not present. It is suggested from these results that pollenitin has a skeleton of 3,5,4'-trihydroxy-7-methoxy-flavone.

Pollenin $a$ and $b$ : Both pigments exhibited absorptions in the region of $359 \mathrm{~m} \mu$ (Band I), the position of which was hypsochromic as compared with that of pollenitin, and the region of $273 \mathrm{~m} \mu$ (Band II) which was the same as pollenitin. It seemed likely that the sugar moiety attached to 3 position. The extensive 
base shift with sodium ethylate indicated that the hydroxyl group of either in 3 or $4^{\prime}$ position was substituted. Moreover, a large bathochromic shift of Band I without a decrease in its relative intensity is a good indication of the presence of a $4^{\prime}$-hydroxyl group. Therefore, the hydroxyl group at $4^{\prime}$-position was believed to be free. Consequently the sugar moiety must be attached to 3 position. The aluminum chloride- and fused sodium acetate shifts indicated the presence of a free hydroxyl group in 5 position and a substituted hydroxyl group in 7 position.

The mass spectrum of pollenitin. The mass spectrum was determined on a Hitachi, RMU-6 type mass spectrometer by the indirect method.* Pollenitin gave the molecular ion at $m / e=316(89 \%$, relative intensity) and $(\mathrm{M}-15)^{+}$ion at $m / e=301(99 \%)$ as the base peak which is corresponding to the loss of the methyl group, namely fission of the methoxyl group. This cracking pattern was observed in the mass spectrum of acacetin (5,7-dihydroxy-4'-methoxyflavone). The ions $m / e=287(23 \%)$ and $m / e=273(37.5 \%)$ arise by loss of carbon monoxide from the ions $m / e=316$ and $m / e=301$, corresponding to each benzofuran cation. Therefore, from the mass spectrum, it was found that the molecular weight of pollenitin is 316 and it seemed to have a methoxyl group.

The NMR spectrum of pollenitin. The trimethylsilyl ether of pollenitin was prepared, treated successively with hexamethyldisilazane and trimetylchlorosilane by the method reported by Mabry et al.5) The NMR spectrum of the trimethylsilyl ether was recorded in

* The MS spectrum was recorded by Dr. A. Tatematsu, Meijo Univ., Nagoya, Japan, to whom the author expresses his thanks.

** The NMR spectrum of the trimethylsilyl ether of pollenitin was recorded by Dr. S. Kuwatsuka, Rikagaku Kenkyu sho, Tokyo, Japan, to whom the author expresses his thanks. carbon tetrachloride with internal tetramethylsilane.** (Japan Electron Optics Lab. Freq. 60 MG). In the resultant spectrum of the trimethylsilyl pollenitin, four proton signals could be assigned to the flavonoid nucleus..$^{-81}$ : a three proton signal at $\delta 3.83$ (singlet) attributable to the protons of the methoxyl group; one proton singlet at $\delta 6.17$ attributable to the single aromatic proton of the 6-position of the A-ring; four displayed typical signal patterns for B-ring protons, that is, $\mathrm{H} 2^{\prime}, 6^{\prime}$, doublet $(J=9 \mathrm{cps})$ at $\delta 7.97(1 \mathrm{H})$ and $8.12(1 \mathrm{H}) ; \mathrm{H} \mathrm{3}^{\prime}, 5^{\prime}$, doublet $(J=9 \mathrm{cps})$ at $\delta 6.78(1 \mathrm{H})$ and $6.93(1 \mathrm{H})$.

Alkaline degradation of pollenitin. About $10 \mathrm{mg}$ of pollenitin and $100 \mathrm{mg}$ potassium hydroxide with one drop of water were fused for an hour at $180^{\circ} \mathrm{C}$. On the other hand, it was refluxed with $20 \%$ pottasium hydroxide solution for an hour at $120^{\circ} \mathrm{C}$. After cooling, the reaction mixtures were diluted with water and acidified with dil. hydrochloric acid, and ethyl ether, and then $n$-buthanol extractions were carried out. The resultant acids and phenols were examined by co-chromatography on paper by using seven different developing solvents and five color reagents. Only $p$-hydroxy benzoic acid derived from the B-ring was identified, but the phenolic substance derived from the A-ring was not identical with such known substances as phlorogrucin, and iretol which was obtained as the alkaline degradation product of wogonin (5,7-dihydroxy-8-methoxyflavone).

Acknowledgement. The author wishes to thank Dr. K. Munakata, Professor of Nagoya University, for his guidance and helpful criticism on the manuscript.

5) T. J. Mabry, T. Kagan and H. Rösler, Phytochemistry, 4, 177 (1965).

6) A. C. Waiss, Jr., R. E. Lundin and D. J. Stern, Tetrahedron Letters, 10, 513, (1964).

7) T. J. Betterham and R.J. Highet, Aust. J. Chem., 17, 428 (1964).

8) B. H. Koeppen and G. G. Roux, Biochem. J., 97, 444 (1965). 